\title{
The Magic Touch: A Case Report of How Smartphone Fingerprint Technology Was Utilized to Establish a Last Known Well Time for Recanalization Treatment in a Patient With Acute Ischemic Stroke
}

\author{
Jessica Choi ${ }^{\mathrm{a}, \mathrm{c}}$, James Rhee ${ }^{\mathrm{b}}$, Maya Lewis ${ }^{\mathrm{b}}$, Shlee Song ${ }^{\mathrm{a}}$
}

\begin{abstract}
Tissue plasminogen activator (tPA) is currently a standard of care for acute stroke patients. One of the necessary criteria in determining eligibility for tPA is the last known well (LKW) time. The LKW time is unfortunately often difficult to obtain accurately if no witness is available, thus posing as an obstacle for acute recanalization therapy. We present the case of a patient who arrived unresponsive with an unwitnessed onset of symptoms concerning for an acute stroke. An LKW time was able to be successfully established by using her fingerprint to unlock her phone and discover a coherent text sent a few hours prior. Patient was able to receive intravenous (IV) tPA and demonstrated remarkable recovery. The use of fingerprint ID to unlock the patient's phone raises the concern of breach of privacy and whether involuntary smartphone searches apply to the emergency code of conduct outlined by the FDA. Smartphone applications, such as Apple iOS "Medical ID" argues for maximal utilization of smartphone technology for emergent medical conditions. Utilization of smartphone technology can potentially serve a potential solution, but the question remains as to whether this practice would be deemed to be ethically appropriate under the policy of implied informed consent under emergent conditions.
\end{abstract}

Keywords: Stroke; Last known well; tPA; Technology; Ethics

\section{Introduction}

Tissue plasminogen activator (tPA) has been the standard of care for acute stroke patients since its approval by the US Food

Manuscript submitted January 29, 2020, accepted February 11, 2020

${ }^{a}$ Department of Neurology, Cedars Sinai Medical Center, Los Angeles, CA, USA

bDepartment of Emergency Medicine, Cedars Sinai Marina del Rey Hospital, Marina del Rey, CA, USA

${ }^{\mathrm{c} C o r r e s p o n d i n g ~ A u t h o r: ~ J e s s i c a ~ C h o i, ~ D e p a r t m e n t ~ o f ~ N e u r o l o g y, ~ C e d a r s ~ S i n a i ~}$ Medical Center, Los Angeles, CA, USA. Email: jessica.choi@cshs.org

doi: https://doi.org/10.14740/jmc3431 and Drug Administration (FDA) in 1996. Inclusion and exclusion criteria for IPA are determined by several factors including recent major surgery, head trauma, anticoagulation use, evidence of hemorrhage on imaging and the last known well (LKW) time. The LKW time is defined as the last time a patient is known to be without signs and symptoms of the current stroke or at his or her baseline state of health. The pioneering NINDS trial (1995) for intravenous (IV) tPA established the time window of $3 \mathrm{~h}$ from the LKW to time of presentation when considered for eligibility [1]; however, the subsequent ECASS III trial (2008) further expanded this window to 4.5 $\mathrm{h}$ [2], and this standard is utilized today. The LKW time is, however, unfortunately often difficult to ascertain and can pose as an obstacle in administering IV tPA. When patients arrive unresponsive or aphasic, and there is no one available to obtain a history from, a clear LKW time cannot be established and thus excludes patients from receiving IV tPA, even if the LKW time may have been within $4.5 \mathrm{~h}$. Other exclusion criteria can be secondarily delineated through the aid of technology, such as lab values (i.e. prothrombin time (PT)/international normalized ratio (INR) and partial thromboplastin time (PTT)) or imaging, but LKW time largely relies on verbal history. We present the case of a patient who arrived unresponsive, alone, and with symptoms concerning for an acute stroke, who was able to have a LKW time established through the aid of her smartphone and thus successfully receive IV tPA.

\section{Case Report}

A 31-year-old woman was brought in by ambulance from the airport after losing consciousness shortly after deplaning an international flight. Upon arrival to the emergency department, patient was minimally interactive and was noted to have left-gaze preference, right hemiplegia and aphasia. Computed tomography (CT) of the head revealed a hyperdense left middle cerebral artery. However, patient's eligibility for tPA was hindered as the LKW time was unknown. Hospital staff came across the patient's smartphone, which was secured with fingerprint technology. In an effort to obtain more information, staff used the patient's finger to unlock the phone and subsequently came across a text message that had been sent by the patient about $2 \mathrm{~h}$ prior, just minutes after landing. The text mes- 
sage was coherent without any syntax or grammatical error, showing that $2 \mathrm{~h}$ prior, the patient had not been aphasic. The time of this text message was subsequently used as the LKW, and the patient was able to receive IV tPA before proceeding to mechanical thrombectomy. Patient was found to have clots in both the middle and anterior cerebral arteries with subsequent successful recanalization. By discharge, patient demonstrated remarkable recovery with minimal residual right-sided weakness and speech issues. Patient was appreciative of emergency room nurses who were able to unlock her phone to obtain critical information for determining patient's tPA eligibility.

\section{Discussion}

Rapid triage and collection of information is critical for stroke patients to ensure adequate therapy within the accepted time window. In the case of our patient, she was able to receive IV tPA but at the potential breach of her privacy. This raises the question as to whether involuntary smartphone searches apply to the same emergency code of conduct outlined by the FDA, which states "planned emergency research involves the systematic investigation of a condition experienced by individuals in a setting where the emergency circumstances require prompt action and generally provide insufficient time and opportunity to locate and obtain consent from each subject's legally authorized representative" (FDA 2013) [3]. While the search of personal property by hospital personnel has been legally upheld countlessly [4], it is unclear whether a locked smartphone would apply to the same standard. In this particular case, further discussion with the risk management division at this specific hospital verified that the benefits outweigh any risks of privacy invasion, so long as the staff keep their viewing of the phone limited only to the information which will assist with the diagnosis and treatment options for the patient. The advent and widespread use of applications, such as Apple iOS "Medical ID" [5], which allows individuals to set up critical medical information to be readily accessible by first responders even via a locked screen, further demonstrate the general public support for the maximal utilization of smartphones during emergency situations without concern for breach of privacy.

\section{Conclusion}

The LKW time is often a barrier for patients in receiving IV tPA if there is insufficient history available. Utilization of smartphone technology (ie. fingerprint identification) can serve as a potential solution, as seen in our patient. However, the question remains as to whether this action is deemed as an ethically approved emergency search under the policy of informed consent, and if this method is one that can be continually utilized for acute, unresponsive stroke patients in determining tPA eligibility.

\section{Acknowledgments}

We thank the hardworking emergency department nursing staff at this hospital in responding astutely during the triage of this case.

\section{Financial Disclosure}

None to declare.

\section{Conflict of Interest}

None to declare.

\section{Informed Consent}

Not applicable.

\section{Author Contributions}

Jessica Choi reviewed the case and gathered the data to create this case report. Shlee Song was the senior author of this manuscript and the vascular neurologist involved in the initial triage of the case. James Rhee was an emergency physician involved in the initial triage of the case. Maya Lewis is a stroke program coordinator and has been involved in setting up the ER nursing care at the involved hospital.

\section{Data Availability}

Authors declare that data supporting the findings of this study are available within the article.

\section{References}

1. The National Institute of Neurological Disorders and Stroke rt-PA Stroke Study Group. Tissue plasminogen activator for acute ischemic stroke. N Engl J Med. 1995;333(24):15811587.

2. Hacke W, Kaste M, Bluhmki E, Brozman M, Davalos A, Guidetti D, Larrue V, et al. Thrombolysis with alteplase 3 to 4.5 hours after acute ischemic stroke. N Engl J Med. 2008;359(13):1317-1329.

3. Exception from informed consent requirements for emergency research. April 2013. Available from: https://www. fda.gov/regulatory-information/search-fda-guidancedocuments/exception-informed-consent-requirementsemergency-research. Accessed October 7, 2019.

4. Health care law monthly. October 2012. Available from: https://www.alston.com/-/media/files/insights/publications/2012/10/searches-of-hospital-patients-their-roomsand-bel/files/ homonthlyoct2012burnette/fileattachment/ hcmonthlyoct2012burnette.pdf. Accessed October 7, 2019.

5. Medical ID. September 19,2019. Available from: https://support.apple.com/en-us/HT207021. Accessed October 7, 2019. 